\title{
Differenzierte Organisationen - die Zukunft für Spitäler
}

Dies ist der abschliessende Teil einer Artikelserie, in der wesentliche Aspekte der Einführung von Fallpauschalen im Jahr 2012 beleuchtet wurden. Es zeigte sich, dass innovative Lösungen, auf den Druck des neuen Vergütungssystems zu reagieren, weiter an der Polarität zwischen Medizin und Management auflaufen. Die «Black Box» der Medizin und die «Tool Box» des Managements finden nicht zueinander. In diesem Beitrag wird gezeigt, dass in den Entwicklungen der Medizin selbst Optimierungen angelegt sind, die sich in differenzierten Organisierungen erschliessen. Darin liegt der Schlüssel.

Christof Schmitz, Peter Berchtold

Korrespondenz: Dr. med. C. Schmitz college $\mathrm{M}$ Freiburgstrasse 41 CH-3010 Bern Tel. 0316323026 Fax 0316323025
Der Entscheid zur Lebertransplantation war für Alfred Koller dann doch eine grössere Belastung, als er anfänglich angenommen hatte. Nicht die Transplantation selber lag ihm auf dem Magen, sondern das Warten, die Ungewissheit und natürlich die wiederkehrenden Fieberschübe. Als ob das nicht schon genug wäre, meldeten sich zwei ältere Geschichten zurück, die er schon fast vergessen hatte. Vor rund 5 bis 6 Jahren war er mit einer depressiven Störung und später wegen erhöhter Blutdruckwerte in Behandlung. Nach einigem Hin und Her und vor allem vielen Gesprächen liess sich Alfred Koller für eines dieser neuen Betreuungsprogramme für Patienten wie ihn motivieren. Sein Hausarzt überzeugte ihn damit, dass dieses Programm spezifisch auf seine Situation zugeschnitten sei, trotzdem war er anfänglich eher skeptisch: alle 3 Wochen einen Termin bei der speziell ausgebildeten Pflegefachperson in der Praxis des Hausarztes und viel Gewicht auf Selbstbehandlung und Selbstkontrolle. Aber schon nach den ersten paar Wochen war er mehr als zufrieden und fühlte sich gut betreut. Die Pflegefachperson war auch ausserhalb der sonst sehr strukturierten Besuche für ihn da, sie und sein Hausarzt, der bei einigen Terminen zugegen war, sprachen sich offensichtlich laufend mit der Hepatologin oder den Chirurgen ab, und immer wieder wurde in den Gesprächen klar, dass Anpassungen in seinem Behandlungsplan aufgrund von Guidelines und Checklisten erfolgten. Gerade kürzlich, an einem gemütlichen Nachtessen mit Freunden, hatte er vertreten, dass dieses differenzierte Miteinander der Behandelnden für ihn ein wesentlicher Faktor für Qualität und Sicherheit sei.

\section{Organisations différenciées: I'avenir des hôpitaux}

Alors que le système SwissDRG va être introduit dans le but d'optimiser les processus et d'améliorer l'efficience - avec le risque que cet objectif ne se réalise pas -, l'avancée des connaissances en médecine offre de véritables moyens d'optimisation. Les multiples possibilités diagnostiques et thérapeutiques de la médecine moderne nécessitent des modes d'organisation différenciée. Seule une médecine qui en tiendra sérieusement compte sera véritablement efficace. La clé est un alignement des systèmes thérapeutiques.

\section{Kongruenz}

Das in Begleitforschungen nachgewiesene Unterbleiben von Prozessoptimierungen unter DRG-Vergütung ist so bemerkenswert wie problematisch. Bemerkenswert ist, dass eine zentrale, zur Einführung des Systems motivierende Annahme nicht zutrifft und das folgenlos bleibt. Problematisch ist, dass Kostendämpfungen wesentlich mit Verdichtung von Arbeit und erhöhter Belastung des medizinischen Personals erreicht werden. Soll mit der Einführung des DRG-Systems in der Schweiz das Gleiche geschehen? Wir haben in den vorherigen drei Artikeln dieser Serie gezeigt, dass Optimierungsversuche in Spitälern nach wie vor an der Polarität zwischen Medizin und Management «kranken». Abgekürzt for- 
muliert, die «Black Box» der Medizin und die «ToolBox» des Managements finden nicht zueinander und produzieren wechselseitig unangemessene Simplifizierungen [1]. Das frustriert und führt zu Abwehrschlachten statt zu innovativen Lösungen. Erst ein Vorgehen, das der Komplexität und der Natur der Medizin gerecht wird, kann chancenreich sein.

Den Anlass für diesbezügliche Innovationen - um den Terminus «Prozessoptimierung» angemessen zu transzendieren - stellt übrigens nur in zweiter Linie die Einführung eines neuen Vergütungssystems dar. Den primären Anlass bildet der enorme Zuwachs an Wissen in der Medizin. Dieses Wachstum ermöglicht heute, mehr als 14000 Krankheiten zu erkennen und viele von ihnen zu behandeln. Zur Behandlung all dieser Krankheiten stehen ein paar tausend medizinische Interventionen und Medikamente zur Ver-
Es braucht eine kongruente Konfiguration der verschiedenen Elemente, die zur wirksamen Behandlung spezifischer Krankheiten nötig sind. Darum geht es.

Kongruente Konfiguration meint differenzierte Systeme. Offensichtlich macht es einen gravierenden Unterschied, ob es um einen einfachen Harnwegsinfekt geht oder eine komplexe Krebserkrankung. Das eine wie das andere benötigt unterschiedliche Organisierungen des erforderlichen Wissens: Grundversorgung im einen, idealerweise ein Comprehensive Cancer Center an einem Universitätsspital im anderen Fall. Die Offensichtlichkeit dieses Unterschieds verstellt den Blick auf die Bedeutung der wirksamen Ausgestaltung kongruenter Organisierungen. $\mathrm{Zu}$ viel ist hier dem historisch Gewachsenen, den üblichen (Spital-)Strukturen mit ihren disziplinären, hierarchischen und kulturellen Differenzlinien überlassen. Mit

\section{Weitverbreitet ist immer noch, möglichst alles mit einem Angebot zu versorgen.}

fügung. Das hat nicht nur ökonomische Folgen, sondern wesentlich auch Konsequenzen für die Organisierungen der Krankenbehandlungen. Das vielfältige medizinische Wissen erfordert vielfältige Prozesse des Organisierens, nur dann kann es in adäquater Weise zur Anwendung kommen. Alles Wissen nützt nichts, wenn nicht Patienten, Kompetenzen, Technologie, adäquate Abläufe usw. aufeinander abgestimmt sind.
Richard Bohmer zu sprechen: «Without deliberate design, healthcare delivery organisations will continue to disappoint [2].»

Spitäler als Ganzes wie auch ihre Ärzte haben sich darauf einzustellen. Weitverbreitet ist immer noch, möglichst alles mit einem Angebot zu versorgen, so wie das traditionell der Fall war. Beispielhaft stehen dafür die Universitätsspitäler, die sich - mit Aufnah-

\section{Abbildung 1}

Elemente eines Behandlungssystems.

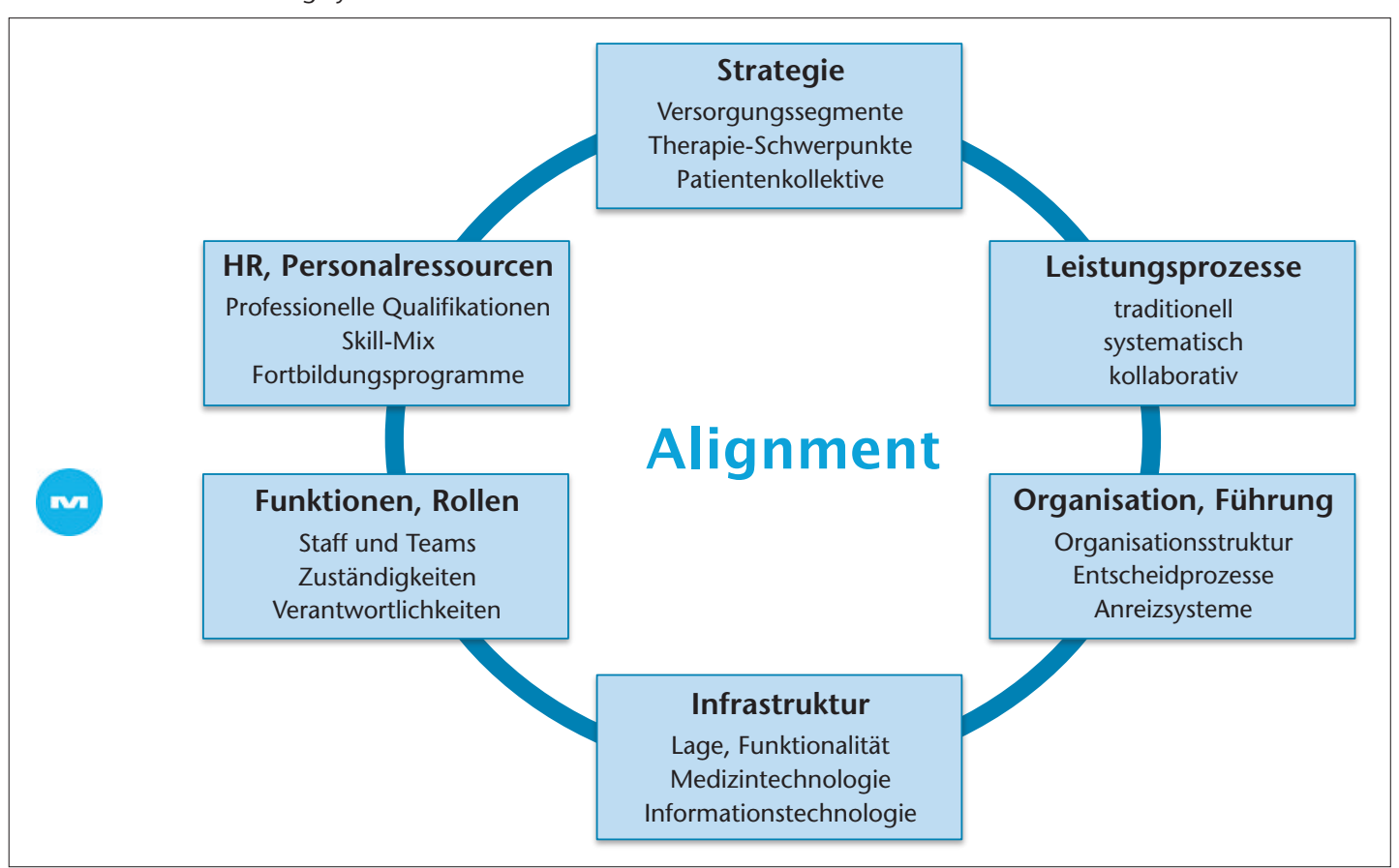


mepflicht argumentierend - für alles vom Schnupfen bis zur komplexesten Transplantation als zuständig verstehen. Bei solcher Bandbreite käme es einem Wunder gleich, würde jede Behandlung effizient geschehen. Viel zu mannigfaltig sind die Organisationserfordernisse der vielen verschiedenen Krankheiten und Patientenprobleme. Das Ergebnis ist ein Auseinanderfallen von Patientenbedürfnissen und Leistungsprozessen. Diese Kluft birgt - der Punkt ist nicht zu unterschätzen - ein gehöriges Frustrationspotential für das Personal. Gleichzeitig hält die hohe Bandbreite des Angebots strategische Unklarheiten darüber aufrecht, wohin die Reise des Spitals gehen soll. Strategische Klarheit wäre aber wichtig, um sich mit der speziell von den Ärzten geforderten Autonomie und Selbstbestimmtheit einbringen zu können. Hier beisst sich die Katze gleich mehrfach in den Schwanz. dieser Abstimmung, in diesem Alignment liegt der Schlüssel zum Erfolg [2].

Die einleitende Sequenz aus der Krankengeschichte von Alfred Koller illustriert ein solches, erfolgreiches Alignment. Für die komplexe Erscheinungsform seines Leidens werden sowohl systematische, wie traditionelle wie kollaborative Formen ins Spiel gebracht. Vorhandenes Wissen wird interdisziplinär und systematisch eingebracht und im Netzwerk der Behandelnden genutzt und immer wieder neu abgestimmt. Das Netzwerk passt sich den Entwicklungen an. Es lernt. All das ist zu organisieren - und es ist so zu organisieren, dass es nicht nur wirksam, sondern auch wirtschaftlich ist. Nicht alles muss das Spital machen, nicht alles muss der Hausarzt selber machen. Unterschiedliche Aufgaben können von unterschiedlichen Akteuren mit unterschiedlichen Qualifikationen und Honorarsätzen wahrgenommen werden.

\section{Es geht um die kritische Prüfung dessen, was ist, und die differenzierte Neuausrichtung auf die Möglichkeiten, die die Medizin heute bietet.}

\section{Alignment als Schlüssel}

Nimmt man diese Erkenntnis - differenzierte medizinische Diagnose- und Therapie-Ansätze benötigen differenzierte Formen des Organisierens - ernst, ist die entscheidende Frage nicht mehr so sehr, wie Ärzte durch finanzielle und andere Anreize dazu gebracht werden können, effizienter zu sein und «einen besseren Job zu machen». Es geht vielmehr um die Frage, wie medizinische Leistungsprozesse so unterschieden und gestaltet werden können, dass die verschiedenen Elemente eines Behandlungssystems, also alle jene für die Behandlung eines Patienten(-kollektivs) nötigen klinischen Leistungen und Entscheidungen, aufeinander abgestimmt und optimiert sind (Abb. 1). In
Wird jeder Akteur zum richtigen Zeitpunkt, gut vorbereitet und eingebunden ins Spiel gebracht, dann hat das Qualität und dann ist das ökonomisch. Das ist Prozessoptimierung (ohne dass man sie als solche ausschildern müsste). Dass solches in mehr Qualität und Patientenzufriedenheit mündet, ist in der letzten Nummer des New England Journal of Medicine 2010 an einem eindrücklichen Beispiel - «Collaborative Care»- nachzulesen [3].

\section{Differenzierungen auf mehreren Ebenen}

Die Medizin auf den Punkt bringen - das vorhandene Wissen adäquat, also differenziert zu organisieren verlangt, die Elemente eines Behandlungssystems zu

\begin{tabular}{|c|c|}
\hline Element & Leitfrage \\
\hline Strategie & $\begin{array}{l}\text { Auf welche Patientenkollektive, Therapieschwerpunkte und Versorgungssegmente zielen wir als } \\
\text { Gesamtspital, als Klinik oder sonstige Einheit? In welcher Kooperation mit wem? }\end{array}$ \\
\hline Leistungsprozesse & $\begin{array}{l}\text { Welche Formen (traditioneller Professionalismus, systematisierte und kollaborative Medizin) } \\
\text { in welcher Mischung betreiben wir in welchen Patientenprozessen? Was wäre besser zu nutzen? }\end{array}$ \\
\hline Organisation & $\begin{array}{l}\text { Welche Organisationsstrukturen, welche (klinischen) Entscheidprozesse und welche Führungs- } \\
\text { systeme unterstützen? Wie beobachten wir Abweichungen wie Erfolge? Wie sorgen wir für } \\
\text { Lernen und Verbesserung? Wie messen wir uns? }\end{array}$ \\
\hline Personalressourcen & $\begin{array}{l}\text { Welche (fachlichen) Qualifikationen, welcher Skill-Mix und welche Entwicklungsmöglichkeiten } \\
\text { sind nötig? }\end{array}$ \\
\hline Funktionen, Rollen & Welches klinische und Support-Staff, welche Zuständigkeiten und Verantwortlichkeiten braucht es? \\
\hline Infrastruktur & $\begin{array}{l}\text { Welche Medizintechnologie braucht es? Wie steht es mit unserer Informationstechnologie? } \\
\text { Welche anderen Geräte, Einrichtungen, räumliche Ressourcen in welcher Ausgestaltung sind } \\
\text { nötig? }\end{array}$ \\
\hline
\end{tabular}


identifizieren und aufeinander abzustimmen. Nicht mehr und nicht weniger. Es sind Entscheidungen darüber zu treffen, welche Patienten(-kollektive), mit welchen Leistungsprozessen, in welcher Organisation, mit welchen Funktionen und Rollen, Staff und Infrastruktur behandelt werden sollen. Das ist, für sich alleine genommen, nichts Revolutionäres. Im Gegenteil zutiefst plausibel ist es, dass z. B. Kompetenzen, Infrastruktur und Steuerungsformen aufeinander bezogen sein müssen, will man erfolgreich Patienten behandeln. Resümiert man etwa die ernsthaften Versuche der letzten Jahre, Zentren zu bilden, kann man an ihrem Gelingen wie Scheitern ablesen, wie Alignment erfolgreich Platz greifen konnte oder eben nicht. Und jeder ernsthafte Beteiligte kann konstatieren, wie anspruchsvoll solche Organisierungen waren und wie viele Lernschritte zu gewärtigen waren. Klinische Wissensflüsse und Entscheidprozesse zu organisieren, liegt rasch quer zu den üblichen und herkömmlichen Funktionen, Territorien, professionellen Selbstverständnissen usw. Nochmals: Keine Revolutionen sind nötig, auch keine völligen Neuerfindungen, sondern konzise, um Kongruenz bemühte Gestaltungsprozesse. Das ist anspruchsvoll genug. Anspruchsvoll darum, weil Alignment bedeutet, selektiver $\mathrm{zu}$ werden, bewusster $\mathrm{zu}$ entscheiden, differenzierter zu beobachten und intensiv für Verbindlichkeiten zu sorgen.

Essentiell ist, dass dieses Abstimmen alle Elemente des Behandlungssystems umfasst. Nötig ist dabei die Differenzierung von sechs Dimensionen (Tab. 1), die übrigens die im letzten Artikel beschriebene Black-Box der Medizin und Tool-Box des Managements miteinander verknüpfen. Der Unterschied, der dadurch entsteht, kann am einfachsten im Vergleich mit den berühmt-berüchtigten «Behandlungspfaden» verdeutlicht werden. Behandlungspfade versuchen im wesentlichen bereits existierende Abläufe aufzuzeichnen und zu «begradigen». Die «eigentlichen Prozesse» sollen abgebildet, anschliessend verbindlich erklärt und eventuell mit ökonomischen Werten unterlegt werden. Das kann ein sinnvolles Vorgehen sein, aber nur dort, wo es sich um stark standardisierbare Behandlungen handelt. Alignment orientiert sich anders. Auch hier spielen Abläufe, Störungen und Effizienz eine Rolle. Aber diese Thematik ist viel stärker im Kontext des Behandlungssystems zu sehen und zu entscheiden, also eingebettet in die Fragen, welche Patienten mit welchen Formen der Medizin, mit welcher Organisation, welchem Personal, in wel- chen Funktionen und Rollen sowie welcher Infrastruktur behandelt werden sollen. Klar, das ist aufwendiger - aber der Natur der Medizin angemessener.

\section{Was ist zu tun?}

Differenzierung spielt sich, das macht das Thema nochmals anspruchsvoll, auf verschiedenen (Versorgungs-)Ebenen ab. Entsprechende Beispiele reichen daher von ganzen Spitälern, etwa dem Istituto Clinico Humanitas in Mailand, das auf seinem Campus drei verschiedene Spitäler, nämlich je eines für Notfall, elektive Eingriffe und Rehabilitation errichtete, weil man der Vermischung der drei unterschiedlichen Anforderungen an Organisation wie Personal begegnen wollte, bis hin zu Beispielen der Behandlung bestimmter Patientensegmente, z. B. chronisch Kranker wie im zitierten Artikel zu «Collaborative Care». Typischerweise geht es beim Letzteren um das konzertierte Zusammenspiel verschiedener Akteure über Organisationsgrenzen hinaus. Ein nächstes, für Anspruch sorgendes Thema. Bereits diese wenigen Hinweise machen klar, dass die Erweiterung der Perspektive von der einfachen (betriebswirtschaftlich orientierten) Optimierung hin zum Alignment einen Komplexitätssprung bedeutet. Einfacher ist das nicht zu haben, denn viel mehr Entscheidungen sind zu treffen. Und genau das ist der Punkt, Entscheidungen sind zu treffen: Welche Patienten(-Kollektive) meinen wir? Mit welchen Prozessen wollen wir diese behandeln? Mit welchen Ressourcen? Mit welcher Steuerung? Mit welchem Staff? Natürlich ergeben sich Einschränkungen der Entscheidungen durch existierende finanzielle, räumliche, personelle und andere Gegebenheiten, die zu berücksichtigen sind. Aber genau darum geht es: kritische Prüfung dessen, was ist, und differenzierte Neuausrichtung auf die Möglichkeiten, die die Medizin heute bietet. Sind die Behandlungssysteme, die wir meinen, kongruent konfiguriert? Mit dieser Frage beginnt die Reise. Richtung Alignment.

\section{Literatur}

1 Berchtold P, Schmitz C. Anatomie der (stationären) Behandlungsprozesse. Schweiz Ärztezeitung. 2010;92(3):84-6.

2 Bohmer R. Designing Care. Harvard Business Press; 2009.

3 Keaton WJ, Lin EHB, Von Korff M et al. Collaborative Care for Patients with Depression and Chronic Illnesses. New Engl J Med. 2010;363(27):2611-20. 